\title{
Questions aux praticiens de la scène
}

Joël Aguet, Bernard Bloch, Eric Eigenmann, Sébastien Grosset, Vincent Laughery, Colin Pahlisch et Stanislas Roquette

\section{OpenEdition}

\section{Journals}

Édition électronique

URL : http://journals.openedition.org/edl/1094

DOI : 10.4000/edl.1094

ISSN : 2296-5084

\section{Éditeur}

Université de Lausanne

\section{Édition imprimée}

Date de publication : 15 mars 2018

Pagination : 183-192

ISBN : 978-2-940331-67-3

ISSN : 0014-2026

\section{Référence électronique}

Joël Aguet, Bernard Bloch, Eric Eigenmann, Sébastien Grosset, Vincent Laughery, Colin Pahlisch et Stanislas Roquette, "Questions aux praticiens de la scène », Études de lettres [En ligne], 1 | 2018, mis en ligne le 15 mars 2020, consulté le 21 décembre 2020. URL : http://journals.openedition.org/edl/ 1094 ; DOl : https://doi.org/10.4000/edl.1094 


\section{QUESTIONS AUX PRATICIENS DE LA SCÈNE}

En conclusion de ce volume, cette dernière partie présente les réponses que certains auteurs et d'autres praticiens de la scène théâtrale ont apportées à trois questions portant sur leurs rapports au théâtre et sur ses liens avec le monde universitaire. Quelle importance a le théâtre? Quels sont les défis du théâtre contemporain? Y a-t-il une problématique qui devrait être traitée d'urgence par les études théâtrales universitaires?

\section{Joël Aguet}

Après un Master en Lettres à l'Université de Lausanne, Joël Aguet alterne programmes de recherches universitaires sur l'histoire du théâtre en Suisse romande ${ }^{1}$ et engagements comme dramaturge auprès de divers metteurs en scène et directeurs de théâtres. Actuellement, il prépare plusieurs ouvrages sur des lieux de mémoires romands du XVII ${ }^{e}$ au XXI ${ }^{e}$ siècle.

Quelle importance a le théatre? L'importance du théâtre est de 10\%. Bien sûr, dans certaines circonstances historiques, franchement peu enviables, l'attraction de la scène a pu compter beaucoup plus pour des peuples opprimés à qui elle offrait des occasions extrêmement courues de rire des dénonciations, même à mots très couverts, des travers de leur société ou de rêver d'ailleurs inaccessibles. Dans notre contexte où le consensus général, pour ne pas dire le système de pensée hégémonique, ne parle

I. Citons notamment Cris et écrits: textes sur le théâtre (1944-1982), Lausanne, Payot, 1990; Dix saisons à la Comédie de Genève, 1989-1999, Genève 1999; Le Carouge 1958-2008: chronique du théatre de Carouge, Carouge, Fondation du Théâtre-Atelier de Genève, 2008, ainsi que les chapitres sur le théâtre dans l'Histoire de la littérature en Suisse romande, ss. la dir. de Roger Francillon, Genève, Zoé, 2015 et le Dictionnaire $d u$ théatre en Suisse, Zurich, Chronos, 2005, pour lequel il responsable de la partie francophone. 
plus que d'individus tout en les envisageant comme des classes de consommateurs plus ou moins bien repus «selon leur mérite», où la règle du moindre effort intellectuel est devenu modèle, on ne s'étonnera pas que la fréquentation occasionnelle des institutions théâtrales comme de toutes leurs marges concerne grosso modo une personne sur dix. C'est en tous cas ce que montrent dans les grandes lignes les rares études statistiques sur le sujet de ces dernières décennies.

Quant à la logique mise en œuvre au bout du Lac, qui, sous prétexte de "répartition des tâches entre communes et canton", attribue au gré des envies des édiles les «secteurs culturels» à l'une ou l'autre de ses instances subventionnantes, elle s'inspire manifestement d'un temps où chaque dame patronnesse avait "ses" pauvres. Ces rivalités sapent les pouvoirs publics. Ceux-ci - tout le monde le sait en Suisse - ne sont efficaces qu'en étant subsidiaires et complémentaires. Chaque niveau politique d'une communauté humaine a le droit de contribuer et de se sentir représenté par l'ensemble des forces culturelles du corps social.

Quels sont les défis du théâtre contemporain? Il s'agit de remettre l'être humain au centre et de lui offrir, par le théâtre, des ouvertures sur de nouvelles dimensions.

$Y$ a-t-il une problématique qui devrait être traitée d'urgence par les études théâtrales universitaires? Par son double travail sur le sens et les sens permettant de mieux comprendre le monde qui nous entoure et de mieux le ressentir, le théâtre doit trouver à l'Université comment s'inscrire au centre de toute procédure pédagogique.

\section{Bernard Bloch}

Bernard Bloch est acteur et metteur en scène. Il a fondé en 1971, à Strasbourg, le Théâtre de la Reprise auquel Denis Guénoun, ami de jeunesse et camarade de pensée, s'est adjoint comme dramaturge et co-metteur en scène. En 1975, il fonde, toujours à Strasbourg et avec Denis Guénoun et Patrick Le Mauff, L'Attroupement, une compagnie qui a profondément marqué la vie théâtrale française de la fin des années 1970. L'Attroupement fut pour Bernard Bloch une expérience déterminante sur le plan artistique et humain, car c'est là que se sont posées les questions qui sont au fondement même de la légitimité du service public. En 1996, il fonde Le Réseau (théâtre), compagnie qu'il dirige encore actuellement et qui est implantée à Montreuil (SeineSaint-Denis) depuis 1999.

Quelle importance a le théâtre? Finalement, à cette question, la seule réponse que je puisse donner ne concerne que moi. Le théâtre est important pour moi puisque j'en vis matériellement et spirituellement 
depuis plus de 50 ans. Il me permet, le temps d'une représentation quand j'en suis le spectateur, ou le temps plus long des répétitions et des représentations quand j'en suis acteur ou metteur en scène, de "créer du réel». Un réel qui n’a d'autres contraintes que celles, spatiales, de la taille de l'aire de jeu ou celles, temporelles, de la durée d'une représentation. Puisse cette invention d'un "autre» réel être partagée par le plus grand nombre de participants! Puissent ces participants être d'origines sociales, ethniques et générationnelles les plus diverses possibles! Et alors, ce partage d'un autre réel que celui qui nous écrase et nous semble le seul possible pourra-t-il peut-être nous permettre de bouger un peu et de ré-entrevoir «l'insoutenable beauté du monde», comme dit Imre Kertész.

Quels sont les défis du théâtre contemporain? Dans une société comme la nôtre qui vit (encore!) en paix et dans une certaine opulence depuis la fin de la Seconde Guerre mondiale, seuls les «non-pauvres» ont accès aux salles de théâtre. Et ce malgré tous les efforts faits depuis 75 ans pour "démocratiser la culture». Que faire pour que ceux qui subissent les injustices et qui devraient constituer le public prioritaire d'un art émancipateur, ne soient pas les grands absents des salles de spectacle?

$Y$ a-t-il une problématique qui devrait être traitée d'urgence par les études théâtrales universitaires? Il me semble que le point aveugle de la recherche théorique est avant tout d'ordre sociologique et politique. Comment faire pour que le théâtre, et l'art en général, puisse devenir l'activité centrale de tous les êtres humains? Comment penser une organisation de la société qui se donnerait pour objectif de permettre à tous et à toutes de faire de leur vie un art? L'autre problématique que l'Université serait bien inspirée de mettre sur le métier est la question de la critique de théâtre. La critique aujourd'hui, notamment en France, se borne le plus souvent, dans le meilleur des cas, à analyser le thème d'un spectacle, la pertinence de son discours. Et dans le pire des cas, le critique nous livre son sentiment de spectateur: c'est bien ou ce n'est pas bien, on s'ennuie ou on s'amuse, les acteurs sont bons ou mauvais. Pour moi, homme de théâtre, acteur, metteur en scène et auteur, j'aimerais que les critiques m'apprennent quelque chose sur ce que j'ai commis. Sur ce qui est abouti ou non dans l'essai que je propose sur le plateau. Je rêverais d'une critique qui participe à ma propre recherche. 


\section{Eric Eigenmann}

Professeur au Département de langue et de littérature françaises modernes à la Faculté des lettres de l'université de Genève, il est responsable de l'Atelier-théâtre du Département de français (ATDF) où il met en scène des spectacles depuis 1997. Il agit occasionnellement comme dramaturge pour des compagnies théâtrales indépendantes à Genève et intervient à la Manufacture (Haute école des arts de la scène à Lausanne).

Quelle importance a le théatre? Le théâtre a toute l'importance que recèle l'art dans les sociétés humaines, exacerbée par le rassemblement réel et vivant des collectivités qu'il implique. Exacerbée, elle l'est en outre tout particulièrement dans une époque qui, comme celle que nous vivons actuellement, tend à privilégier l'économie d'une part, les contacts virtuels entre les humains d'autre part.

Quels sont les défis du théâtre contemporain? Parmi les nombreux défis que relève le théâtre contemporain, défis qu'évoque ma réponse à la question précédente, distinguons celui-ci: le théâtre contemporain doit s'attacher à rester perpétuellement... contemporain, ce qui suppose de ne pas oublier le passé.

$Y$ a-t-il une problématique qui devrait être traitée d'urgence par les études théâtrales universitaires? Dans la foulée encore de ce qui précède se noue la problématique suivante: comment le contemporain scénique se fabrique-t-il à partir de son histoire, socio-politique, philosophique et esthétique? Mais il faudrait aussi traiter des fonctions que les études théâtrales universitaires exercent déjà dans le domaine des activités théâtrales en général, du côté de la production comme du côté de la réception, et qu'elles pourraient développer.

\section{Sébastien Grosset}

Assistant à l'EPFL (École Polytechnique Fédérale de Lausanne), à la Faculté de l'environnement naturel, architectural et construit (ENAC), dans l'Atelier de la Conception de l'Espace (ALICE). Il enseigne ponctuellement à la HEAD (Haute Ecole d'Art et Design à Genève) et prépare une thèse sur l'architecture d'intérieur en philosophie à l'Université de Lausanne. Depuis quelques années, il est auteur dramatique et dramaturge.

Quelle importance a le théâtre? Si je m'intéresse aujourd'hui à l'architecture, c'est parce que je pense qu'elle a objectivement plus d'importance que le théâtre. Il m'a hélas été donné une fois ou deux de participer à des spectacles qui ne tenaient pas debout - expériences pénibles, mais qui n'occasionnèrent ni procès ni décès. S’il s'était 
agi de bâtiments, il en aurait été tout autrement. Larchitecture a plus d'importance que le théâtre parce que son degré de participation au monde est plus élevé. C'est ce qu'il y a à la fois de stimulant et parfois d'inquiétant dans cet art que l'on enseigne dans les écoles polytechniques. Ceci étant, c'est peut-être précisément dans sa moindre importance que réside l'intérêt du théâtre. Moindre importance par rapport à l'architecture, mais aussi, semble-t-il, à d'autres arts qui paraissent avoir plus de prise sur le monde. Pour de nombreuses raisons (dont plusieurs que j'ignore), le théâtre semble avoir rejoint l'opéra, la céramique et l'escrime artistique dans un certain éloignement de la marche du monde. Or, il n'est pas impossible que cette distance avec le cours des choses ménage comme un espace de contemplation, une "pause de participation", selon le mot de Lambert Wiesing. Du point de vue du créateur, c'est l'occasion d'expériences: on peut prendre plus de risques lorsque ce qu'on fait n'a pas beaucoup d'importance.

Quels sont les défis du théatre contemporain? Il se peut que certains défis qui se posent au théâtre aujourd'hui se jouent dans cet entre-deux. Je suppose (c'est un point autour duquel je ne fais encore que tourner) qu'il y a un lien entre l'ambiguïté de cet art de la fiction en live et un problème dramaturgique qui me taraude depuis de nombreuses années, l'expression de la tension entre la linéarité du temps de nos vies et la désorientation constitutive de l'espace contemporain. Je pense à l'espace architectural, mais aussi à celui, plus abstrait, des réseaux. La narration linéaire ne rend certes plus compte de la spatialisation de notre temporalité contemporaine: nous sommes ici et là en même temps et circulons dans des réseaux mondialisés au point que la compréhension du déroulement de nos occupations relève plus de la géographie que de l'histoire. La dramaturgie se doit sans doute d'exprimer cet éclatement temporel et c'est un défi qu'elle relève. Mais si elle se contente d'imiter la structure des réseaux, elle prend le risque de ne plus rendre compte de notre temporalité individuelle qui elle, reste très classique: nous avons un début et une fin. Si je dois choisir un défi à relever, ce sera donc celui de l'expression conjointe d'un monde qui se parcourt sous la forme non linéaire du réseau infini et de nos vies qui, elles, demeurent envers et contre tout vouées à une finitude certaine.

$Y$ a-t-il une problématique qui devrait être traitée d'urgence par les études théâtrales universitaires? Je ne me risquerai pas à suggérer une problématique aux études théâtrales, mais peut-être une attitude: 
aborder le théâtre avant tout comme une chose. C'est, je crois, dans l'ambiguïté qu'il entretient avec sa dimension concrète que le théâtre a peut-être l'occasion de faire entendre sa voix dans le concert des arts. La réalité de la scène, physiquement, est la même que celle de la salle: un lieu, des objets et des gens sont là, devant leurs spectateurs, et ce n'est que par convention qu'ils acquièrent un statut spécifique. Il n'en va pas de même au cinéma ou en littérature où l'on a face à soi un écran ou une page qui sont des espaces physiquement impénétrables. Ce n'est que de façon métaphorique qu'on peut dire qu'on «entre dans une histoire». Aborder le théâtre dans ce qu'il a de concret, c'est garder vivant le mystère de la distance volontaire que ses spectateurs entretiennent avec lui. C'est une façon, aussi, de le situer par rapport aux autres arts de la scène. Ce qui en effet caractérise à mes yeux (mais cela n'engage que moi) le théâtre par rapport à la performance ou au concert, c'est la fiction. On peut prendre l'énoncé dans l'autre sens et dire que dès lors que la scène accueille de la fiction, il s'agit de théâtre. Pour le dire en franglais, le théâtre, d'abord, c'est de la fiction en live. C'est là que réside à mes yeux sa spécificité, mais aussi son ambiguïté - et peut-être, tout compte fait, son importance.

\section{Vincent Laughery}

Doctorant FNS à l'Université de Lausanne (section d'anglais), il écrit une thèse sur la métaphore et l'agentivité dans les tragédies de Shakespeare. Il participe également à la vie théâtrale de l'Université comme acteur et metteur en sène.

Quelle importance a le théâtre? Connaissez-vous la blague du maçon portugais qui vient toquer à la porte d'une ménagère? «Bonjour Madame Doupont, jé viens pour faire la mour!» Et le malheureux d'être giflé pour son obscénité involontaire. Grand moment de théâtre de boulevard. Le théâtre met en relation des corps dans divers états, exprimant des tensions de part et d'autre du "quatrième mur». Ce mur, comme on l'appelle un peu tristement, parfois avec ironie, est lui aussi générateur de tensions spécifiquement théâtrales, étant donné que la scène comme la salle collaborent à l'ériger ou à le briser. Ainsi, le théâtre est un lieu de présentation: «Bonjour, Mesdames et Messieurs, nous venons pour faire la mour. Quatre fois». Or, une présence se négocie constamment avec une absence et un dédoublement. Corps de l'actrice, corps d'un personnage: celui qui est là tend à s'effacer pour faire voir l'autre qui n'est pas là, à part dans la fiction dramatique. Et entre le théâtre et le 
drame, l'espace présent et celui qu'on imagine, est-ce la même relation? Qu'est-ce que le théâtre apporte au drame, auquel on a accès aussi par la littérature? Faisons sans personnages et sans drame, on verra bien ce qui reste... Mais c'est que ce sont là des absences persistantes: elles sont toujours bien là, surtout lorsqu'on les chasse, tout comme le "quatrième mur", tout comme la confusion "le mur/l'amour", tout comme la perversité perçue du maçon... Entre chien et loup : présences qui veulent s'effacer, absences qui prennent corps dès qu'il y a des corps, même non humains... Le théâtre, c'est donc ce qui vivote, papillonne entre le théâtre et le drame, entre comédienne et personnage, maçon et pervers, entre chien et loup. Si pour vous, les maçons sont pervers, les portugais obscènes et l'humour machiste, allez au théâtre où votre chien sera le loup d'un-e autre. Où d'autre qu'au théâtre nous arrive-t-il de ressentir si inébranlablement l'incertitude, l'inquiétude même, de ne pas savoir si c'est un chien qu'on a vu, ou un loup...?

Quels sont les défis du théatre contemporain? Je comprends "contemporain" au sens général d' «actuel» et "théâtre contemporain" comme regroupant aussi le théâtre universitaire. Le théâtre se pratique beaucoup à l'Unil et à l'EPFL et c'est tant mieux. Les troupes universitaires ne subissent pas comme les professionnelles la pression de vendre leurs spectacles, avec tout ce que cela peut impliquer comme compromis. Pourquoi, dès lors, ne pas en profiter pour expérimenter, s'essayer à des esthétiques diverses? Les études offrent une phase propice à lancer des projets, tester des idées ou envies, tenter des paris fous sur scène tout en sachant que le spectacle trouvera son public. Le théâtre universitaire paraît hautement capable de meubler une scène pendant 1h30-2h de manière originale. Or, cette capacité est double: d'une part, c'est paradoxalement un manque de formation aux métiers du théâtre dont on peut faire une force considérable: sans mode d'emploi, on va monter son meuble différemment. Moins commode, moins bien monté, monté complètement faux... Ce n'est pas grave, faisons du faux (il n'y a que ça de vrai au théâtre)! Ce sera une création tout à fait surprenante, défiant certaines conventions (les ignorant) de manière habituellement impensable à moins d'une solide expérience. La qualité ne sera pas la même, cela ne fait rien à l'affaire: tant que le public est diverti, un spectacle qui hésite entre deux genres est potentiellement tout aussi complexe qu'une mise en scène aboutie qui les associe harmonieusement. D'autre part, une capacité positive: quelle que soit sa faculté, on peut 
amener de sa formation en cours non seulement des questions qui se prêtent à l'exploration au théâtre, mais aussi une certaine approche à ces questions, sans rien mentionner de la faune de fantasmes et d'espoirs qui sommeille au fond de ces questionnements. Une pièce montée par des chimistes et des sociologues ensemble, c'est la guerre ouverte! Sûrement... mais sur scène, c'est juste une histoire de couple. Bricolons à l'Université des spectacles en composant chacune et chacun avec ce qu'on a entre les mains (une statistique, un roman, trois quilles, une partition...) et assumons le défi d'un théâtre expérimental! De toute façon, ce n'est pas comme si on savait faire du théâtre qui ne l'est pas...

\section{Colin Pahlisch}

Assistant en section de français à l'Université de Lausanne, il travaille sur la question de la Communauté dans la littérature de science-fiction contemporaine. Il est l'auteur de la pièce de théâtre Mer qu'il a mise en scène à La Grange de Dorigny et ailleurs avec le soutien des Maîtres de la Caverne.

Quelle importance a le théâtre? Il s'agit à mon sens de l'une des premières formes de création dont nous avons gardé la trace dans l'histoire de l'humanité. Le théâtre revêt ainsi un rôle fondamental au niveau anthropologique, sociologique, philosophique et poétique. L'évolution des formes dramatiques accompagnent, reflètent et renseignent en tout temps la pensée humaine. Elles permettent d'approfondir ses questionnements les plus existentiels, de diversifier et d'enrichir nos modes de rapport au monde.

Quels sont les défis du théâtre contemporain? Explorer le potentiel de genres peu représentés. Mieux comprendre nos types d'interaction avec le théâtre. Qu'est-ce qu'être spectateur? Qu'est-ce que lire le théâtre? Développer les rapports entre théâtre et société, drame et critique sociale (voir à cet égard le Théâtre de l'Opprimé d'Augusto Boal).

$Y$ a-t-il une problématique qui devrait être traitée d'urgence par les études théâtrales universitaires? La question des conditions du théâtre constitue selon moi une piste novatrice et stimulante pour les études à venir. Au niveau philosophique, la question des rapports entre théâtralité et interactions sociales à l'ère des réseaux virtuels - engendrant la multiplication des mises en scène de soi via les nouvelles technologies de représentation, ainsi que la diversification des usages et enjeux liés à cette tendance, tant dans la sphère intime que professionnelle - et des humanités numériques, me semble de première nécessité. 


\section{Stanislas Roquette}

Titulaire d'une maîtrise en sciences politiques, Stanislas Roquette est comédien, metteur en scène et enseignant à Sciences-Po Paris et Paris-Dauphine dans le cadre d'ateliers de pratique théâtrale et de prise de parole en public. Il est aussi pédagogue dans des contextes très différents (écoles de théâtre, universités, lycées, collèges, entreprises, groupes amateurs). Acteur notamment pour Jacques Lassalle et Christian Schiaretti, il a mis en scène Les Règles du savoir-vivre dans la société moderne de Jean-Luc Lagarce, en Russie et en russe. En 2008, il a créé avec le mathématicien Miquel Oliu Barton et Denis Guénoun la compagnie Artépo dont il est, depuis 2017, le co-directeur avec Anne-Sophie Dupoux. En variant sans cesse les formes de collaboration entre eux, leur recherche a donné lieu à des propositions multiples. À chaque fois, il s'est agi d'une mise en œuvre physique et scénique d'un travail (ou d'un jeu) de pensée. Lors des dernières années, une "co-conception" des spectacles a souvent prévalu, avec deux points de vue bien distincts: conception depuis la salle pour Denis Guénoun, et conception depuis le plateau pour Stanislas Roquette.

Quelle importance a le théatre? Si je considère que l'importance est l'intérêt porté à quelque chose, et que cet intérêt est toujours relatif à d'autres, il me semble que l'importance du théâtre est toujours faible. Par rapport à l'histoire, à la géopolitique, aux religions, aux sciences, à l'économie, à la sociologie, à la politique, à la psychologie, à la littérature ou à la philosophie, le théâtre n'a que peu d'importance. Outre les gens de théâtre, qui s'en soucie plus que l'on se soucierait d'un luxe, c'està-dire d'une chose parfois belle et exigeante mais cantonnée à l'espace privé du divertissement ou de la culture personnelle? Si je ne parle que de mon expérience, le théâtre a bien sûr beaucoup d'importance. En effet, il constitue l'élément central de ma vie; je ne saurais pas bien quoi faire d'autre. Le théâtre a été pour moi la possibilité de m'ouvrir aux autres, tout en affirmant quelque chose. Envoyer et recevoir, circulation de la vie. De manière générale, je crois aussi qu'il est le moyen absolu pour échanger de manière sensible et vivante sur tous les domaines que j'ai cités: histoire, sciences, littérature, etc. La présence réelle des corps sur un plateau, chargés de transmettre des récits, des idées, des débats ou des émotions, transforme la scène en espace physique et mental où se jouent nos vies, nos contradictions et notre finitude.

Pour ma part, je cherche toujours à rattacher ce que je fais sur scène à une nécessité intime, dont j'espère qu'elle touchera le public par son universalité sans jamais en être tout à fait certain. C'est la pratique du jeu qui me fait toucher ce besoin de théâtre. Dans les ateliers de théâtre que je dirige en France et à l'étranger, à destination de publics de tous âges, de toutes provenances et de toutes conditions sociales, je suis 
toujours frappé par ce que permet le théâtre: un échange universel sur des questions fondamentales, interrogations qui au fond ne varient pas tellement d'un groupe à l'autre. Il s'agit toujours de désirs et de peurs, de conscience du corps et des émotions, de présence à soi-même et au jeu. Le travail au plateau permet une compréhension mutuelle à la fois profonde et instantanée, grâce à la communication non-verbale, audelà donc de la barrière des langues. Faudrait-il que tout le monde se frotte dès le plus jeune âge à la matière théâtrale (dans une salle ou sur une scène, devant un livre ou en atelier) pour en goûter adulte l'infinie richesse? Ce qui est sûr pour moi, c'est que le théâtre en lui-même et pour lui-même n'a aucun sens. Il ne vaut que parce qu'il est tramé par tout ce qui n'est pas lui-même, disons: la vie. En cela, il nous renvoie à notre condition essentielle: le fait d'être traversé par des émotions, des désirs, des visions.

Quels sont les défis du théâtre contemporain? De mon point de vue, le théâtre qui se donne pour mission de dénoncer l'état catastrophique du monde, avec une posture au mieux descriptive, au pire cynique et nihiliste, ce théâtre-là manque désormais son but s'il souhaite éveiller la conscience des spectateurs. La noirceur du tableau de notre époque est déjà tellement présente partout dans la rue et les médias que je rêve d'un théâtre qui chercherait plutôt l'ouverture de nouveaux chemins possibles, la joie d'utopies à partager ou en tout cas, la mise à nu des forces vives à l'œuvre en chacun de nous. Un défi pour le théâtre contemporain serait de ne pas se laisser étouffer par la sinistrose, ni galvaniser par la jubilation morbide et la complaisance dans l'avilissement. Il s'agirait alors par exemple d'exalter l'imagination comme force politique de premier ordre, ainsi que le propose Olivier Neveux.

$Y$ a-t-il une problématique qui devrait être traitée d'urgence par les études théâtrales universitaires? Je ne sais pas, je ne les connais pas suffisamment. La seule chose que je peux dire, c'est qu'il faudrait absolument proposer plus de pratique théâtrale dans les cursus universitaires, avec de véritables praticiens de la scène, de la même manière qu'il faudrait rajouter de véritables enseignements de réflexion dans les écoles d'acteurs. L'entremêlement entre réflexion et pratique, ou entre pensée et jeu, est tellement au cœur de ce que fait le théâtre qu'on ne devrait jamais totalement séparer les deux, il me semble. Les réalisations théâtrales comme les ouvrages de théorie sur le théâtre pourraient beaucoup y gagner. 